\title{
A microprocessor-based real-time BASIC laboratory: A pulse motor-controlled visual stimulator
}

\author{
NAOYUKI OSAKA \\ Otemon-Gakuin University, Ibaraki, Osaka, 567 Japan
}

\begin{abstract}
Using the Commodore PET as a random pulse generator, an inexpensive visual stimulator for psychophysical experiments can easily be made at low cost. The PIA interface that connects the external experimental devices is easily accessed by PEEKing and POKEing its registers with BASIC. Using pulse motors, two independent stimulator wheels, having 24 different color filters and a $2.4 \mathrm{log}(.1 \mathrm{log}$ unit steps) unit range light attenuator, are controlled by a randomized string of pulses via a PIA port, and the responses of the observer as well as the stimulus parameters are automatically written onto the cassette memory for further data analysis.
\end{abstract}

The recent development of microprocessor-based technology (Milkman, Shapley, \& Schick, 1978; Perera, 1978; Polson, 1978; Santa \& Streit, 1978; Sidowski, 1978) has led to the experimental use of real-time laboratory computer systems that are inexpensive.

The recent trends in microcomputer uses in psy. chology are covered elsewhere (Sidowski, 1978).

Input/output (I/O) ports and interfaces for standard keyboards, CRT displays, and data file cassettes are now available at low cost for most microcomputers (Brown \& Deffenbacher, 1978). However, more important has been the development of the capability of the microcomputer to communicate with the outside world, that is, laboratory interfaces for connecting switch inputs and outputs to power drivers. Although a oneboard microprocessor coupled with a peripheral interface adaptor (PIA) with hexadecimal key inputs may be sufficient for real-time control of a visual stimulator (Brown \& Deffenbacher, 1978; Kashinsky, Romanczyk, \& Treiber, 1979) and data acquisition (Criswell \& Babcock, 1978), the BASIC interpreter provided with the Commodore PET appears to be powerful for realtime laboratory experimentation (Durrett, 1978).

Using the PET 2001-8 (6502 CPU-based family with 8K RAM, 14K ROM, and 6-MHz/9-in. black-and-white CRT with 73 key inputs) as a random pulse generator (Osaka, 1978), a fully automated versatile random stimulator was designed for visual psychophysical experiments. The PET has BASIC accessible 8-bit parallel I/O ports for laboratory interfacing: The TTLcompatible pins (PA0 to PA7, CA1, and CB2) of the powerful MOS6522 PIA (VIA) remain free as the user port and are directly BASIC accessible using "PEEKing" and "POKEing" at each $\mathrm{I} / \mathrm{O}$ register address (Commodore Business Machines, Inc., 1978, Note 1; Johnson, 1978; Yob, 1979).

Supported in part by Research Grant 371038 from the Japan Ministry of Education.

\section{INTERFACING HARDWARE}

The hardware logic is shown schematically in Figure 1.
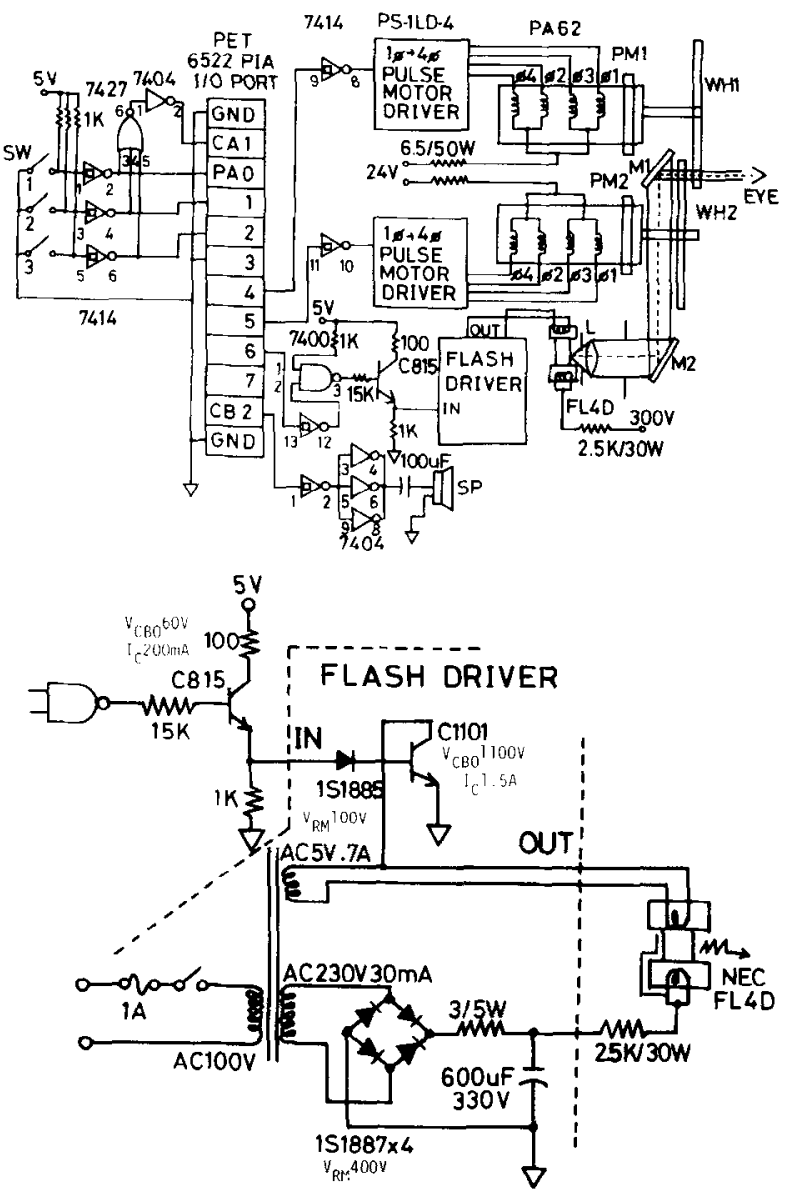

Figure 1. Interfacing logic for the visual stimulator. The PET communicates with the outside devices via a 6522 PIA 1/0 port (inputs: CA1, PA0, PA1, PA2; outputs: CB2, PA4, PA5, PA6). $P M=$ pulse motor, $W H=$ color and ND wheels, $M=$ mirror, $L=$ lens, $S P=$ speaker. 


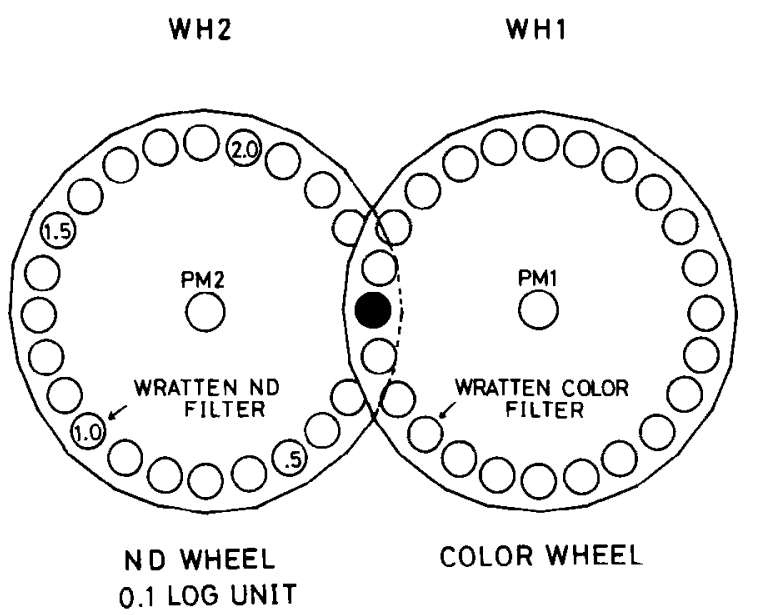

FRONT VIEW

Figure 2. Front view of the stimulator. A randomized combination of the apertures of the color and ND wheels can be made using PET's randomized string of pulses.
Interfacing to the PET is through the MOS6522 PIA port that yields 8 bits information exchange, the direction of which can be set under BASIC control, decimal address 59459 in the PET's memory map (McLean, 1978). In Figure 1, PA0, PA1, and PA2 ports of the PIA are each defined as input modes, whereas PA4, PA5, and PA6 ports are each defined as output modes. The TTL-level logic signal of each $\mathrm{I} / \mathrm{O}$ port is buffered with Schmitt trigger action in order to isolate (protect) the PET's PIA from all of the external device lines.

Pushing (grounding) Microswitch 1 or 2 causes "yes" and "no" response input (logic "1") to the PAO and PA1 ports, respectively. This response is scanned and latched by the PET's program (PEEK statement). Pushing Microswitch 3 initiates a "try-again" routine. Each microswitch is pulled up to $\mathrm{Vcc}(+5 \mathrm{~V})$ by a $1 \mathrm{~K}$ resistor, and each response signal is OR-gated to CAl for input synchronization. The output logic pulse controls two independent pulse motors (Nippon Pulse Motor, Type PA62, and associated driver, Type PS-1LD-
$10 \operatorname{DIM} \times(50), Y(50): ? " c I r "$

20 ?"REAL-TIME BASIC LAB AUTOMIZATION"

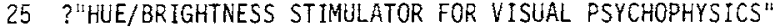

30 GOSUB500:GOSUB550

$40 \quad W=W+1$ : GOSUB 400

50 FORI $=1$ TOHR:X(I) $=I:$ NEXTI

55 FORJ $=1$ TOBR:Y $(J)=J:$ NEXTJ

$60 \mathrm{~N}=0$

70 GOSUB700

$80 \quad \mathrm{~N}=\mathrm{N}+1$

$90 \quad I F N=$ TRGOTO110

100 G0T070

110 ?

120 IFW $=S G 0 T 0140$

130 GOTO40

140 GOSUB 1450

150 CLOSE

160 END

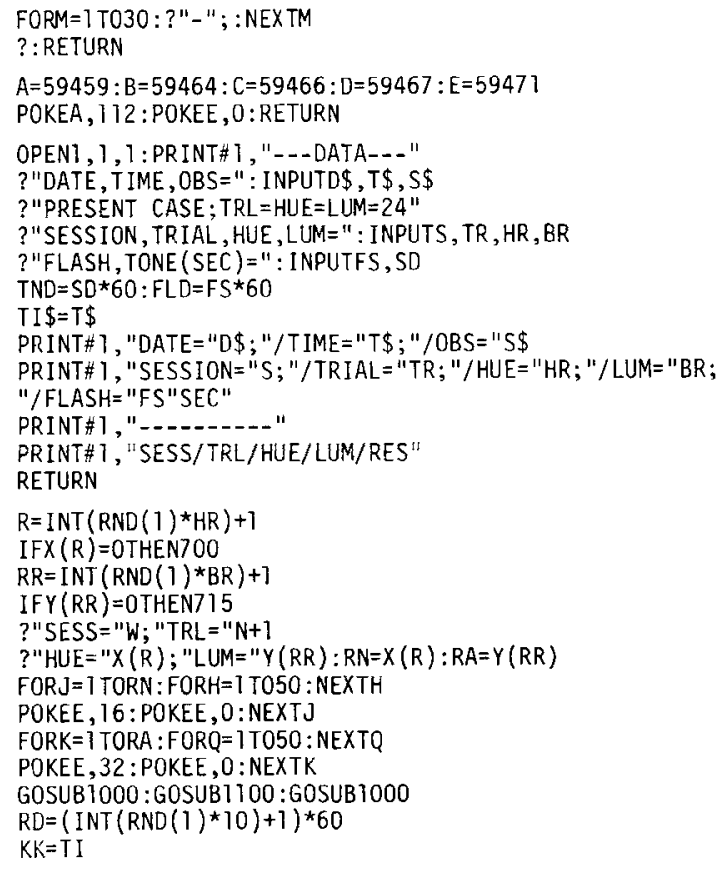

1460 RETURN

IFTI -KK >RDTHEN780

GOTO775

-GOSUB1200:60StB1 300

FRS $=4$ THEN760

GOSUB 1400

REM PULSE MOTOR REWIND (24-STEPS)

$Q=24-R N$

FORYH $=1$ TOQ:FORZ $=1$ TO20:NEXTZ

POKEE, 16 :POKEE, 0 : NEX TYH

$X(R)=0$

$V X=24-R A$

FORXB $=1$ TOVX:FORZZ = 1TO20:NEXTZZ

POKEE, 32:POKEE, 0:NEXTXB

$Y(R R)=0$

IFT I-TM $>120$ THEN 1030

G0T01010

POKED, 16 : POKEB , 123 : POKEC , 151

$\mathrm{KK}=\mathrm{TI}$

IFTI - KK>TNDTHEN] 140

GOT01 120

POKED, 0

?TAB (8) "TONE= "SD" SEC"

$F D=T I$

IFTI-FD>FLDTHEN1240

TER

RETURN

$F=T I$

PEEK (E)AND 7

IFRS $=1$ THEN1370

IFRS $=2$ THENI 380

GOTO1305

?TAB (30)"MISS= "RS : RETURN

?TAB (30)" YES="RS: RETURN

?TAB (30)" NO="RS:RETURN

?TAB (20) "TRY-AGAIN" : ?:RETURN

PRINT\# $1, W ; N ; X(R) ; Y(R R) ; R S$

RETURN

Figure 3. Sample program for the visual stimulator. 
$4),{ }^{1}$ high-voltage flash driver $(+300 \mathrm{~V}$, Type FL4D tube), and a warning sound generator ( $1 \mathrm{kHz} ; 8-\mathrm{ohm}$ speaker). Since an output pulse from PA4 port rotates PM1 for a 15-deg step angle, 24 pulses result in one rotation (360 deg) of an associated color wheel (WH1). Similarly, PA5 port controls PM2 and, accordingly, the neutral density (ND) wheel (WH2). As shown in Figure 2, both wheels have 24 independent apertures $(1 \mathrm{~cm}$ in diameter) so that a maximum of 24 (luminance levels) by 24 (hue levels) stimulus combinations can be made using the PET's randomized string of pulses. As the front view of the wheel shows, Kodak Wratten neutral density filters (.1 log unit steps) and Kodak Wratten color filters control $2.4 \mathrm{log}$ units range luminance and 24 different hues in a random order. A simple optical path leads the light from the source tube to the observer's eye through the lens, the mirrors, and the apertures.

\section{PROGRAMMING}

Figure 3 provides an example BASIC program for the visual stimulator. The program consists of the main program (Lines 10-160) and five associated subroutines (Lines 400-910 and 1450-1460). Furthermore, an additional five nested subroutines (Lines 1000-1410) are each called by associated subroutines (Lines 700-910) as required. The program has several main components.

The main loop (Lines 40-130) is indexed by variables $\mathrm{W}$ and $\mathrm{N}$, which count the numbers of the session and trial, respectively. Each subroutine (Lines 400-510) draws a line on the CRT and selects five decimal addresses $^{2}$ associated with different $\mathrm{I} / \mathrm{O}$ functions in the PET. Since Data Lines PA0-PA7 are individually programmed to function for input or output as required, the user ports of PA4, PA5, and PA6 $\left(2^{4}+2^{5}+2^{6}=\right.$ 112) are defined as output; the other ports remain as inputs. This is done by using a POKE 59459 statement to place a number into the data direction register.

The subroutines (Lines 550-640 and 1400-1460) control the cassette filing functions: The cassette is opened (Line 550), and the experimental parameters (such as the date, time in HHMMSS format, observer, session, trial, hue, luminance, and durations) are stored and written (PRINT\#1 statement) onto the cassette. When the INPUT command is interpreted by the monitor the PET prints "?"3 on the CRT, asking the experimenter to input the variables (or string-\$-mixed) variable) from keyboard. On Line 585, the duration of the flash and tone are converted into a second-based unit, since the PET's software timer indexed by TI (see Line 772) uses a 1/60-sec crystal-oscillated time base. It appears that this most powerful real-time BASIC function depends on the TI function.

The subroutine beginning on Line 700 generates a randomized string of pulses without replacement (randomized within a session: Lines 700-730) and controls the magnitude of the rotation angle of the pulse motors

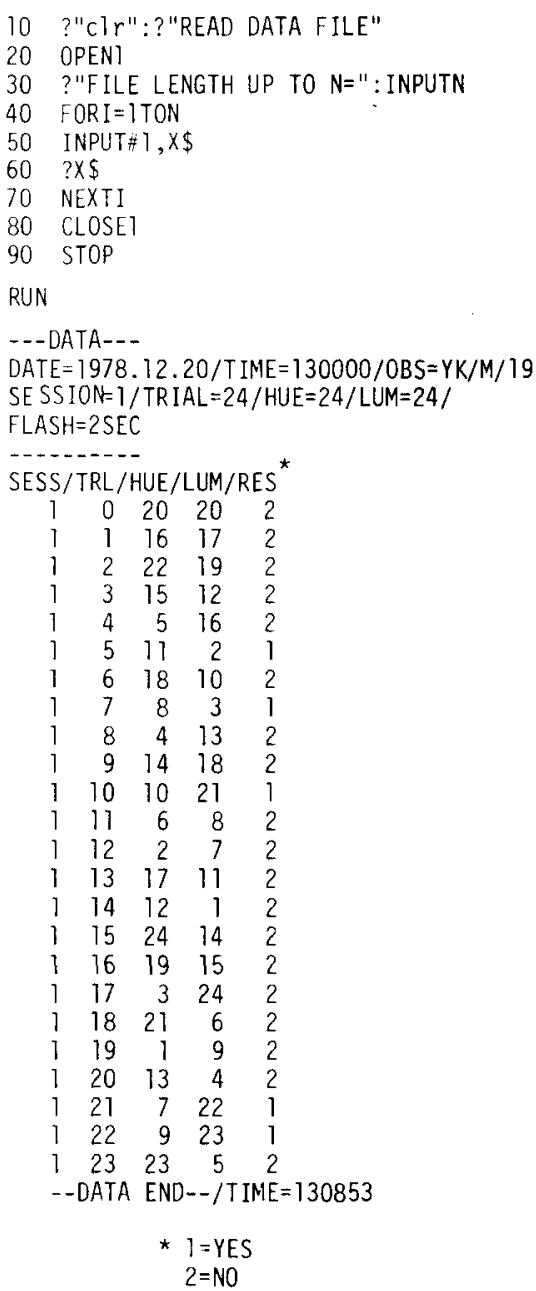

Figure 4. Sample program for reading-out from the data cassette.

(Lines 740-755). The subroutines (Lines 1000-1030 and 1100-1160) delay time for $2 \mathrm{sec}$ in a loop (Lines 1010 1020 ) and trigger the sound signal (as a programmed $1-\mathrm{kHz}$ square wave: Line 1100) for a programmed duration (at Line 580). After the randomized foreperiod time, which changes from 3 to $14 \mathrm{sec}$ (Lines 760-778), a flash stimulates the observer's eye for a given time (Lines 580 and 1200-1250). Immediately after the offset of the flash, a call to the subroutine at Line 780 repetitively checks for the observer's responses for a maximum of $5 \mathrm{sec}$ : PA0-2 $\left(2^{0}+2^{1}+2^{2}=7\right)$ input ports of the PIA are consulted with a PEEK command (accessing these ports using logical AND function), and the response is printed on the CRT (Lines 1365 1390). Pushing Switch 3 causes a "try-again" loop to Line 760.

A call to the subroutine at Line 800 stores the session number $(W)$, trial number $(N)$, level of hue $[X(R)]$ and of luminance $[Y(R)]$, and responses into the RAM area for the cassette buffer until a block of data is ready to be written. Data are not double buffered, so that processing ceases while the block is written, 
and it is difficult to predict exactly when the recording action will occur (McLean, 1978). Lines 810-910 set the angle of the pulse motors to initial position.

The subroutine on Line 1450 writes the data-end remark and the finishing time of the experimental job onto the cassette tape. Following these processes, the cassette file is closed (Line 150).

Figure 4 shows an example program that reads the data from the cassette file and prints it on the CRT for further data analysis. Two minor difficulties have been found during cassette handling and processing. First, as has been previously noted by McLean (1978), the program/data loading procedure has not always been successful. Second, the zero-page RAM sometimes malfunctions. These minor difficulties may be related, in part, to mechanical problems with the cassette unit and to the insufficient heat-sink problems with the RAM.

The stimulus parameters, as well as the response values, are printed on the CRT immediately after the action, informing the experimenter of the progress of the experiment. The use of this system in the animal psychophysics laboratory, for example, will make it possible to execute a hue/brightness discrimination experiment over a few days. The total interface cost of the visual stimulator is less than $\$ 180$.

\section{REFERENCE NOTE}

1. Commodore Business Machines, Inc. MCS6522 versatile interface adaptor. Preliminary data sheet, November 1977.

\section{REFERENCES}

Brown, E. L., \& Deffenbacher, K. Microcomputers big and little: Selecting a low-cost laboratory computer network. Behavior Research Methods \& Instrumentation, 1978, 10, 241-245.

Commodore Business Machines, Inc. PET communication with the outside world. Palo Alto, Calif: Author, 1978.

Criswell, H. E., \& Baвcock, B. A flexible low cost signal averager. Electroencephalography \& Clinical Neurophysiology, $1978,45,535-537$.

Durrets, H. J., JR. Inexpensive microcomputer systems for research and instruction: A dream or reality? Behavior Research Methods \& Instrumentation, 1978, 10, 345-351.

Johnson, R. N. Using the PET microcomputer. Behavior Research Methods \& Instrumentation, 1978, 10, 474-475.

Kashinsky, W. M., Romanczyk, R. G., \& Treiber, A. The presentation of visual stimuli: An inexpensive microcomputerbased system. Behavior Research Methods \& Instrumentation, 1979, 11, 64-66.

McLean, R. S. The Commodore PET: Using personal computers for experimental control. Behavior Research Methods \& Instrumentation, 1978, 10,468-473.

Milkman, N., Shapley, R., \& Schick, G. Experimental applications: A microcomputer-based visual stimulator. Behavior Research Methods \& Instrumentation, 1978, 10, 539-545.

Osaka, N. An inexpensive quasirandom interpulse interval generator. Behavior Research Methods \& Instrumentation, $1978,10,18-20$.

Perera, T. B. A versatile microcomputer-based multiple-field tachistoscope. Behavior Research Methods \& Instrumentation, 1978, 10, 546-547.

Polson, P. G. Microprocessors: Their impact on real-time computing in psychology. Behavior Research Methods \& Instrumentation, 1978, 10, 463-467.

SANTA, J. L., \& Streit, P. A. A microprocessor reaction time laboratory. Behavior Research Methods \& Instrumentation, $1978,10,352-355$.

Sidowski, J. B. Microcomputers in psychology. Behavior Research Methods \& Instrumentation, 1978, 10, 463-467.

Yoв, G. PET user cookbook. Kilobaud, 1979, 27, 62-73.

\section{NOTES}

1. For information on pulse motors contact Nippon Pulse Motor, Inc, 2-16-13 Hongo, Bunkyo-ku, Tokyo 112, Japan [Phone: (03)-813-8841]. Its total cost is approximately $\$ 160$ with associated drivers.

2. A practical $1 / 0$ selection and associated addresses in the PET are: $59459=$ data direction register, $59464=$ timer 2, $59466=$ serial $\mathrm{I} / \mathrm{O}$ shift register, $59467=$ auxiliary control register, $59468=$ peripheral control register, $59471=$ output register without handshaking.

3. A mark "?" is interpreted as an abbreviated command of "PRINT" by the PET BASIC.

(Received for publication May 8, 1979; revision accepted August 14, 1979.) 